\title{
ABM-GIS simulation for urban freight distribution of perishable food
}

\author{
Hanane EL Raoui ${ }^{1,2}$, Mustapha Oudani ${ }^{2}$ and Ahmed EL Hilali Alaoui ${ }^{1}$ \\ ${ }^{1}$ Modeling and scientific computing laboratory, Sidi Mohamed Ben Abdellah University, Morocco \\ ${ }^{2}$ Université Internationale de Rabat, FIL, TIC lab, Morocco
}

\begin{abstract}
Freight transport is essential to modern urban civilization. No urban area could exist without a powerful freight transport system. However, the distribution of perishable foods in urban areas is seen as a source of problems, due to traffic congestion, time pressures, and environmental impact. In this paper, an Agent-Based Model integrated with Geographic Information Systems (ABM-GIS) is designed for a time-dependent vehicle routing problem with time windows. This simulation model consists of determining the quickest routes to transport fresh products, estimating Vehicle kilometer traveled VKT and vehicle hour traveled VHT where speeds and travel times depend on the time of the day. Based on a case study, analyses of changes on traffic condition were conducted to get an insight into the impact of these changes on cost, service quality represented by the respect of time windows, and carbon emissions. The results reveal that traffic jams and restrictive time windows lead to additional cost, cause delays, and increase co2 emission. As for a short-term planning, time-dependent scheduling algorithm was proposed and assessed while extending time windows. Results have proved the potential saving in cost, travel time, and carbon emission.
\end{abstract}

\section{Introduction}

Freight transport is fundamental to modern urban civilization. No urban area could exist without an efficient freight transport system. Considering the demand for high-quality fresh food, transportation requirements for fresh food delivery have been continually increasing in urban areas [1].

The delivery of these goods is perceived as a source of problems. This is owing to specific characteristics of perishable foods, traffic congestion, the increasing requirement of customers in terms of delivery time, and environmental impact. Traffic growth presents a new challenge for carriers in vehicle routing and scheduling to deliver products. Besides, it brings environmental problems due to the increase in carbon emission. Therefore, establishing the fastest routes, optimal departure from the distribution center to deliver these time-sensitive products is a major problem encountered by carriers.

This study focused on a time-dependent vehicle routing problem with time windows for distributing perishable foods in urban areas. Vehicle routing problem (VRP) had a spatial dimension which is practically neglected in research studies. Therefore, handling geographic data is requisite for efficient routes based on real distances. The most promising solution for so is GIS. In fact, we propose an Agent-Based simulation Model integrated with the Geographic Information System (ABM-GIS) to use real-case while performing distances between customers. In this paper, we aim at producing the fastest routes to deliver perishable foods as fast as possible and estimating VHT and VKT which are valuable for transportation, through the simulation model. We aim also to study the impact of congestion on commercial vehicle tours in an urban area. And as for short-term planning, for daily operations, we propose a time-dependent scheduling approach to optimize departure times from the distribution center.

The remainder of this paper is organized as follows: section 2 provides a background on integrating ABM and GIS, section 3 describes the simulation model, section 4 presents the estimation of VKT and VHT, section 5 highlights the impact of congestion, section 6 presents experimental design and results, section 7 introduces the proposed scheduling methods as well as the enhancement they provide, and we end up with a conclusion.

\section{Agent Based Model and GIS integration}

Agent-based modeling is a relatively new method compared to system dynamics and discrete event modeling. This modeling paradigm is developed to simulate complex systems through the study of active entities behavior, known as agents. Agent Based System (ABS) has been adopted to solve complex problems, from various domains, such as logistics optimization, traffic, and urban planning. ABS can be used for different purpose: (1) Understanding observed dynamics, processes, and systems, (2) Designing or engineering of processes or systems, (3) Managing a system or process,(4) Formulating theory and explanatory

acorresponding author: hanane.elraoui@usmba.ac.ma 
models,(5) Predicting, (6) Optimizing resources, capabilities, and processes. For further details refer to [2]. Geographic Information Systems (GIS) are recognized as one of the new technologies which can be usefully introduced to Decision Support System (DSS) for vehicle routing [3]. GIS is well defined as an information technology which stores, analyzes, and displays both spatial and non-spatial data.

Combining these two techniques provides us with the advantages of both and allows a better modeling of the vehicle routing problem which is also a spatial problem.

\section{Simulation model}

\subsection{Case study description}

This paper presents an agent-based model of a food service distributor that provides perishable foods to restaurants, associated with GIS map of Casablanca, one of the most crowded cities in Morocco, where the Distribution Center (DC) and different customers are located.

\subsection{Modelling environment}

For our modeling environment, we use AnyLogic software, Version 8.2 developed by XJTechnologies. The advantage of this software is its ability to support different simulation methodologies, including discrete event simulation, system dynamics and agent-based simulation, in which we are interested. Anylogic has an integrated GIS space where we can drag different objects (agents) and define their properties.

\subsection{Agents}

In our model, agents represent the distribution center, customers, and vehicles used for delivering. After generating agents, the setting is defined in order to make agents live in the GIS Space.

Locations of agents (DC, customers) were defined in a database to let Anylogic place it on the GIS map, through sending the address to an open street map server to obtain the corresponding coordinates.

\subsection{Vehicle controlling}

In the developing model, we use a state chart to provide refrigerated vehicle movement. Anylogic state chart consists of a sequence of states and transitions that enable the user to define the behavior of an object.

Figure 1, 2 and 3 illustrate the state charts used to control vehicles movements during the simulation of different scenarios presented below.

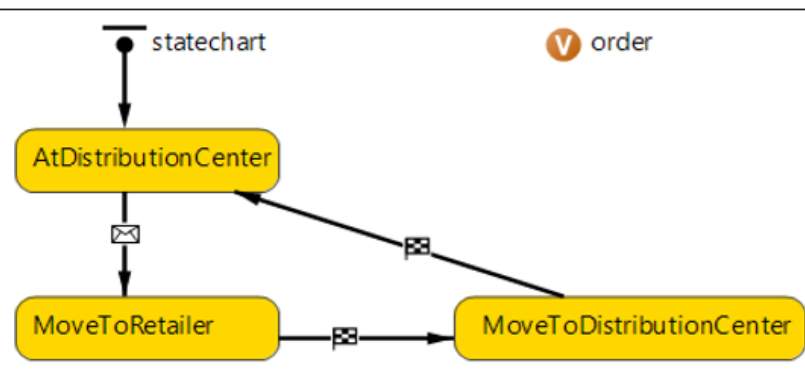

Figure 1.State chart of vehicle in scenario A

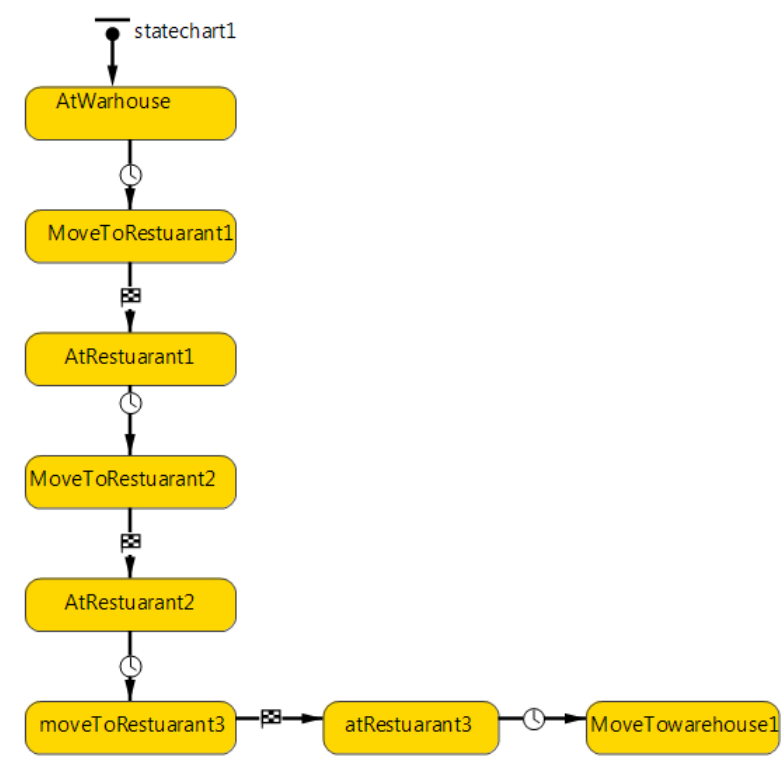

Figure 2. State chart of vehicles in scenarioB -Tour 1

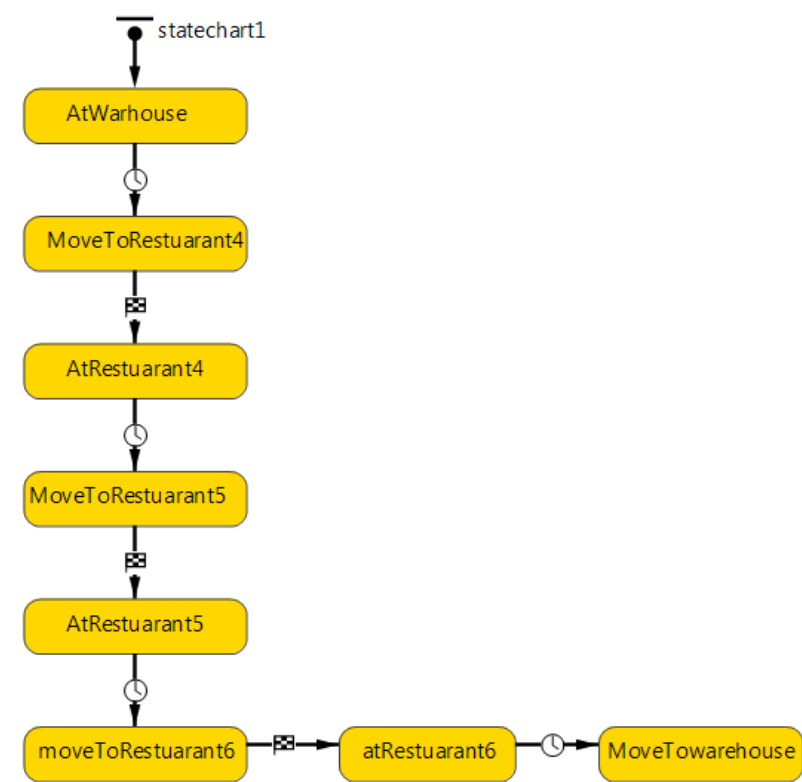

Figure 3. Statechart of vehicles in scenarioB -Tour 2

\section{5 scenarios simulated}

In our study case, we simulate two scenarios corresponding to two types of tours. Within scenario A, the customer order is equal to the capacity of the truck, otherwise known as a Full TruckLoad (FTL). It's a degenerated type of VRP since the vehicle will visit only one customer before returning to the DC.

In scenario B, the customer's order is relatively small and the delivery is Less Than Truckload (LTL). 


\subsection{Routes}

During the distribution of frozen, refrigerated, and fresh goods it may be important to minimize the travel time, because these products have a short shelf life and their quality decay with the variation of temperature. And since keeping the adequate temperature adds significant costs, selecting efficient fastest routes would consequently reduce the whole transportation costs.

To find a suitable path for distributing perishable foods, several techniques have been applied in the literature. however, the shortfall of most researches is that authors apply mathematical equations instead of using spatial data for efficient routes. For example, [4] undertook the fresh meat distribution problem by applying several algorithms to find optimal sets of routes.

To reach customers in time, real route network should be considered, hence the interest of GIS that was adopted by [5] to determine optimal transportation routes. In [6] authors apply GIS to transportation to find optimal roads among sets of routes. To identify the best routes for waste collection, [7] developed an application of ArcGIS Network Analyst tool. In view of this, we opt for GIS in our study.

In the first scenario, customers get delivered directly. Routes that connect the distribution center to each customer are established by using GIS map route on Anylogic. Since we are dealing with time-sensitive products that should be delivered as fast as possible, we choose the fastest routing method option in the routing properties.

In the second scenario, customers are subdivided into balanced tours determined according to this criterion: (1) tours have the same number of stops and (2) the service areas are the same across tours [8].
In both tours, each node is linked to the nearest one through the fastest path set up by the same technique used in the first scenario.

\section{Estimation of VKT and VHT}

In transport planning, it's necessary to estimate the vehicle kilometer traveled by a vehicle to satisfy customer demand as well as the travel time VHT. These two elements are important for carriers to get an insight into variable costs. Furthermore, VHT is valuable for scheduling vehicles departure from the DC.

In the literature, there are several works that deal with the approximation of VKT. In a typical work [9] proposes a continuous approximation to estimate VKT for a Capacitated Vehicle Routing Problem (CVRP). In [10] authors Combine simulations with linear regressions to estimate the length of Traveling Salesman Problem (TSP). In [11] they have used the same techniques in addition to neural networks for an accurate approximation. Most of the previous research, authors compute the VHT and VKT based on direct links. However, in reality, vehicles travel on a real road network. Thus, the Euclidean distances used is far from accuracy and cannot hold anymore. This fact prompts us to adopt GIS function to approximate based on a real road network

After building the AB-GIS model, a simulation run was performed on anylogic software to estimate the VKT and VHT for both scenarios corresponding to FTL and LTL. We assume that roads are not congested, and then the vehicle travel with the speed limit in each arc of the tour. Travel speed limit can be found by open street browser www.openstreetbrowser.orgReferences. And based on statistic outputs about the model execution in the $\log$ file, VHT and VKT are measured. Results are shown in figure 4 and figure 5.

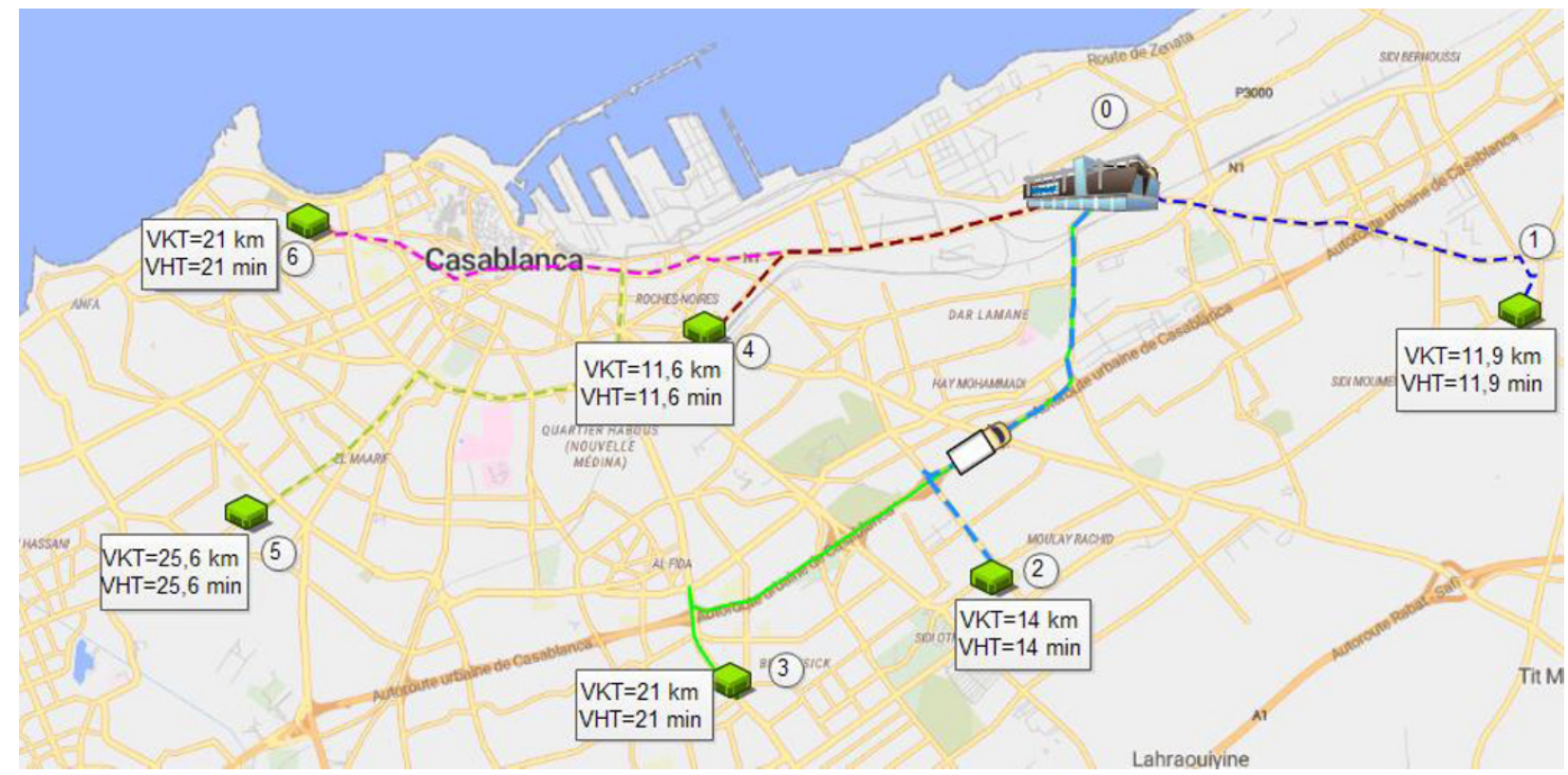

Figure 4.VKT and VHT in scenario A 


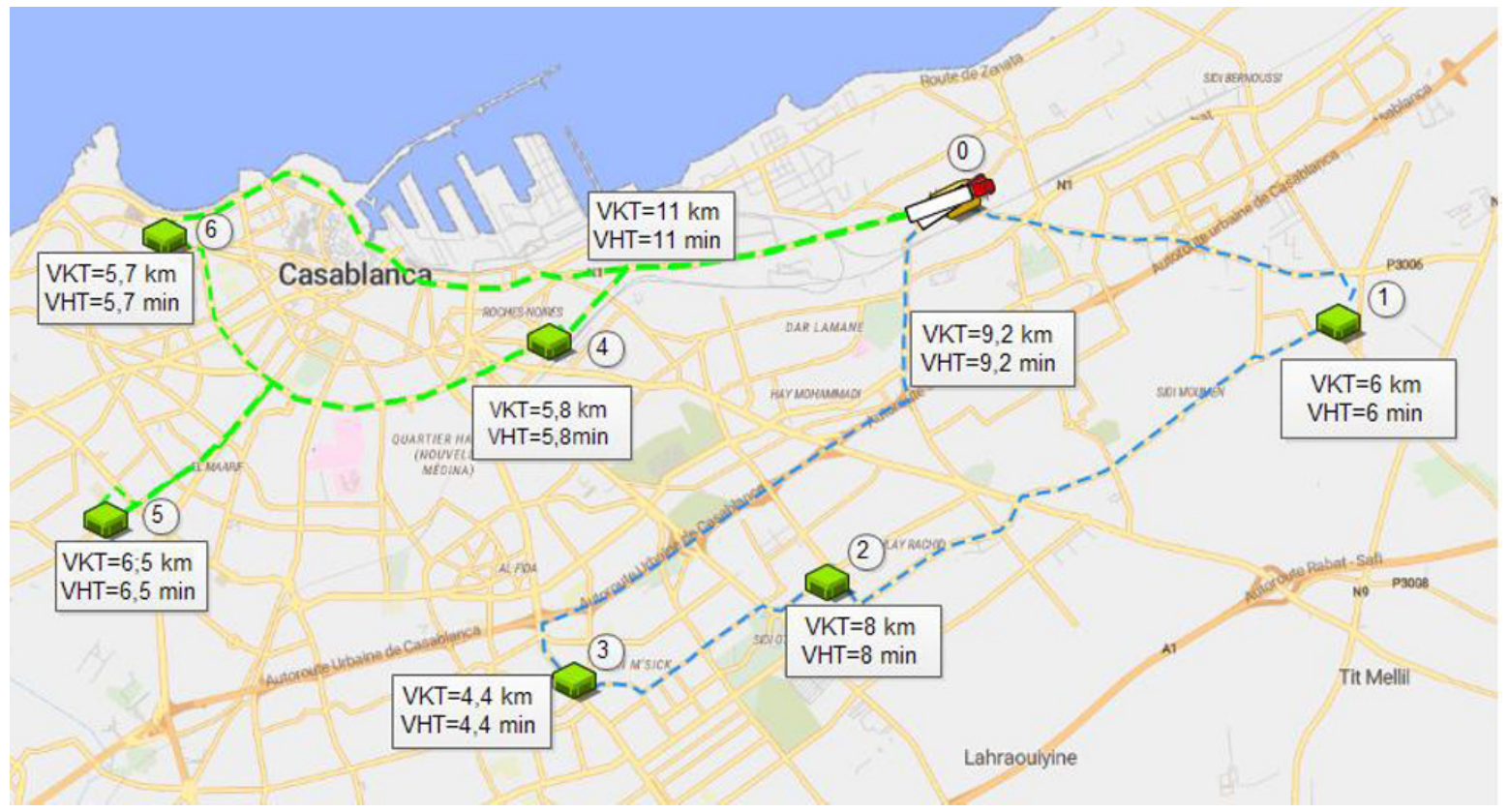

Figure 5.VHT and VKT in scenario B

\section{Study of congestion impact}

\subsection{Impact of congestion on VHT}

Nowadays, customers' requirement for fresh food delivery conditions is increasing in urban areas. Customers have become more exigent about the delivery time; they require to be delivered within a specific time window.

In urban areas, the traffic flow is growing steadily, which makes the delivery of fresh food in time seen as a problem, and this is mostly due to traffic congestion.

Traffic jams may be reached during different times of the day, therefore the delivery time will also depend on the time of the day. However, in today's planning systems, the time dependency is not recognized, and the speed is assumed to be constant. The travel time estimation on those bases cannot be accurate, which may lead to additional costs, customer frustration and penalties due to the violation of time windows.

In this paper the variation of speed during the travel time due to the congestion will be considered, thus we are dealing with a time-dependent travel speed. Our study is based on the distribution of speed shown in figure 6 .
To each time interval corresponds a congestion level represented by a coefficient $\mathrm{C}_{\mathrm{t}}$ computed as:

$$
C_{t}=\frac{S_{t}}{S_{l}}
$$

Where $S_{t}$ the average speed at time t, and $S_{l}$ the speed limit. We assume that the congestion level is the same on all arcs.

\subsubsection{Impact of congestion in scenario $A$}

Before evaluating the impact of road congestion on travel time, we start by getting an insight into the possibility of serving different customers in time under normal conditions. Thus, we calculate the speed required on each arc using the moveToInTime() anylogic function that makes the agent move to a destination in a specified duration. To evaluate how these changes on road condition may affect the VHT, and the service quality represented by the respect of the time window, we test 2 scenarios:

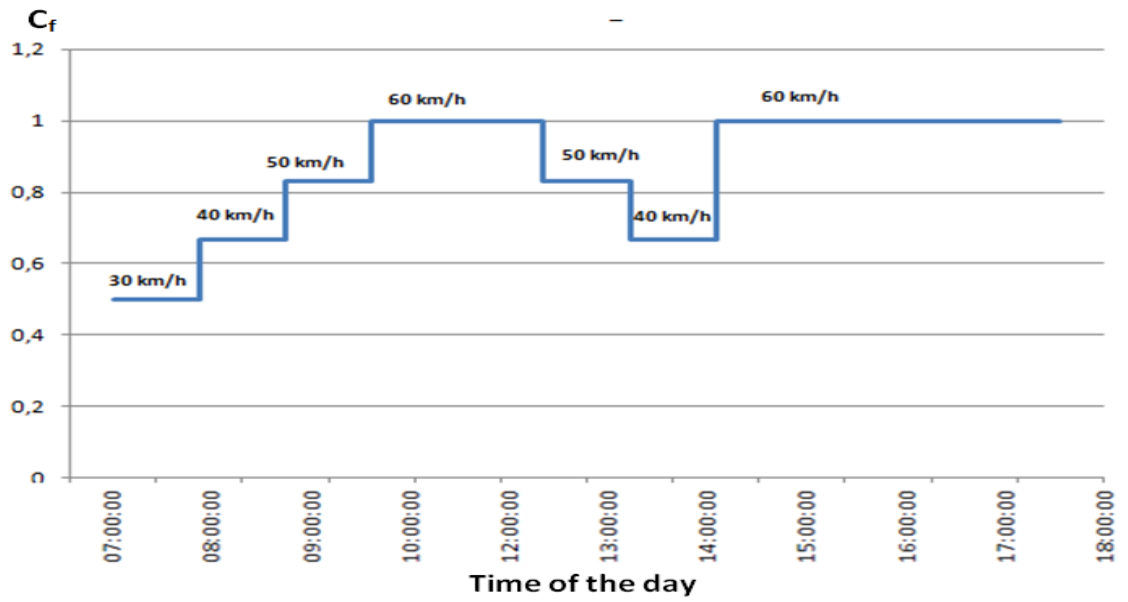

Figure 6. speed distribution 
- Scenario 1:

- Customer Demand is received during a traffic peak (rush hour).

- The speed required to deliver this customer in time lower than or equal to the average speed during the traffic peak interval.

- Scenario 2:

- Customer Demand is received during a traffic peak (rush hour).

- The speed required to deliver this customer in time is higher than the average speed during the traffic peak interval.

For an accurate estimation of the VHT, speed changes within the travel time are taken into account. Two cases are considered, in the first case the departure and arrival time of vehicle are in the same time interval $[\mathrm{K}]$, thus no speed changes have occurred. In the second case when the arrival time of a vehicle is estimated to be in the interval $[\mathrm{K}+1]$ it means the speed has changed during this period.

1: If $D_{0}$ and $A T_{n} \in[K]$

$2: V H T_{0}^{n}=\frac{V K T_{0}^{n}}{V_{k}}$

3: Else

$4: V H T_{0}^{n}=\stackrel{K}{K} \underset{0}{n}+\stackrel{K+1}{V H} T_{0}^{n}$

$5: V H T_{0}^{n}=\left(T_{k \sup }-D_{0}\right)+\left(A T_{n}-T_{k \text { sup }}\right) \times \frac{V_{k}}{V_{k+1}}$

\subsubsection{Impact of congestion in scenario $B$}

In this scenario customers have the same time windows form 8 to 9 in the morning, because customers are restaurants and this time demand ensures that they can process and serve fresh food to their customers.

\subsection{Impact of congestion on costs}

To provide insight into the relationship between cost, and changes in the road network, the distribution costs considered in this study include the transportation costs, damage costs, refrigeration, and penalty costs. Since we are interested in measuring the variation, fixed cost will not be included.

\subsubsection{Transportation costs}

The transport costs include maintenance and repair costs, tires and depreciation costs, and the major component related to the fuel consumption. Fuel consumption, in our study, is weight and time-dependent, because the travel speed and travel time, which depend on departure time, and the loading weight, are taken into consideration in the procedure.

Fuel consumption calculation:
Let $K P L_{i j}$ be the kilometer per liter for a vehicle traveling from the customer $\mathrm{i}$ to customer $\mathrm{j}$. The consumption per unit time corresponding $L P H_{i j}$ is calculated as follow:

$$
L P H_{i j}=\frac{V_{i j}}{K P L_{i j}}
$$

$V_{i j}$ Is the corresponding traveling speed form $\mathrm{i}$ to $\mathrm{j}$.

The transportation costs can be expressed as

$C_{\text {tran }}=\sum_{v=1}^{m} \sum_{i=0}^{n} \sum_{j=0}^{n}\left(C_{i j}^{v} x_{i j}^{v} d_{i j}+C^{\prime v}{ }_{i j}^{v} x_{i j}^{v} V H T_{i j}^{v}\right)$

$C_{i j}^{v}$ Is the sum of variable costs dependent onthe distance traveled (maintenance, tires, depreciation)

$x_{i j}^{v}$ Is a $0-1$ variable, $x_{i j}^{v}=1$ if the vehicle $\mathrm{v}$ passes the road between customer $\mathrm{i}$ and customer $\mathrm{j}$, otherwise 0 .

$d_{i j}$ Distance traveled from node $\mathrm{i}$ to $\mathrm{j}$

$V H T_{i j}^{v}$ Vehicle hour traveled from $\mathrm{i}$ to $\mathrm{j}$

$C^{\prime, v}$ Fuel consumption by unit time of the vehicle $\mathrm{v}$ moving from node $\mathrm{i}$ to $\mathrm{j}$

\subsubsection{The refrigeration costs}

Refrigeration is crucial during the transportation of perishable food. Refrigeration costs include the cost caused by energy consumption to keep the adequate temperature during delivery, as well as the cost of additional energy supplied by the refrigeration system during the unloading process.

Refrigeration cost during transportation can be expressed as:

$$
\begin{aligned}
& C_{T}=c_{e} \sum_{v=1}^{m} \sum_{i=0}^{n} \sum_{j=0}^{n} x_{i j}^{v} T_{i j}^{v} \\
& T_{i j}^{v}=V H T_{i j}^{v}+t^{\prime}{ }_{j}
\end{aligned}
$$

Ce: The refrigeration costs during the transportation process of unit time.

$T_{i j}^{v}$ :The traveling time of the refrigerated truck $\mathrm{v}$ from the customer $\mathrm{i}$ to the customer $\mathrm{j}$.

$t^{\prime}{ }_{j}$ :The waiting time of refrigerated vehicle in customer $\mathrm{j}$ before unloading.

Refrigeration cost during the unloading process:

$$
\begin{aligned}
& C_{u}=c_{e} \sum_{v=1}^{m} \sum_{j=0}^{n} y_{j}^{v_{j}} U_{j} \\
& C_{\text {ref }}=C_{T}+C_{u}
\end{aligned}
$$


$c_{e}^{\prime}$ : The refrigeration costs during the unloading process of unit time.

$U_{j}$ : The unloading time which is needed toserve the customer i.

$y_{j}^{v}$ is a $0-1$ variable, $y_{j}^{v}=1$ if the vehicle $\mathrm{k}$ services for customer i, otherwise $y_{j}^{v}=0$.

\subsubsection{Penalty cost}

Generally, in the distribution of food, customers require to be delivered within a time window. And if the goods do not reach the destination within the time agreed on by the customer, thus the time window is violated and additional penalty cost will be applied. These costs can be expressed as follow:

$C_{p}=\sum_{v=1}^{m} \sum_{j=0}^{n}\left(\alpha \max \left[T w_{\text {inf }}-A T_{j}^{v}, 0\right]+\beta \max \left[A T_{j}^{v}-T w_{\text {sup }}, 0\right]\right)(8)$

$A T_{j}^{v}$ : The arrival time of vehicle v to customer $\mathrm{j}$.

$T w_{\text {inf }}, T w_{\text {sup }}:$ The lower and upper bound of the time window.

$\alpha$ : The cost of waiting for the unit time if refrigerated truck arrives at a customer in advance.

$\beta$ : The cost of punishing for the unit time if the refrigerated truck is late to the customer.

\subsection{Impact of congestion on $c 02$ emission}

The traffic growth in urban areas brings further problems of environmental aspects. In recent years there has been increasing interest in estimating the environmental effects of vehicle routing policies.

In our work, we aim to discover the relationship between traffic congestion, restrictive time windows, and co2 emission. For the purpose, we use the "ASIF" equation [12] to quantify carbon emissions in the simulated scenarios.

\section{Experimental design and results}

In this section, we use a numerical example to quantify the impact of traffic jams on the VHT, costs, and emissions. Section 6.1 describes the problem and parameter setting; experimental results are analyzed in section 6.2.

\section{1 experimental design and parameter settings}

The delivery service provider places a premium on service quality, hence all scenario use hard time windows, to guarantee that promised delivery times would be met.

To simplify the problem, we make the following assumptions:
(1) The service provider has a homogenous fleet with one type of refrigerated vehicles.

(2) In the scenario A, the customer order does not exceed the capacity vehicle, and the time spent to serve each customer is $15 \mathrm{~min}$.

(3) In the scenario $B$, the vehicle can serve 3 customers and spend $10 \mathrm{~min}$ at each one.

The distribution center DC start operating at 7.In the first scenario corresponding to direct delivery, orders in the simulation model are generated as an event sent to the distribution center randomly during the day. Since we are interested in evaluating the impact of congestion we took a sample of orders received during the morning, characterized by the higher congestion level. We assume that order preparation requires $30 \mathrm{~min}$ and the time windows length is $15 \mathrm{~min}$.

In the second scenario, the time window is [8h $9 \mathrm{~h}$, since the level of congestion from 7 to 8 is upper, simulation run was performed to estimate the travel time for both tours with an average speed corresponding to this time window figure to determine if this time interval is sufficient for delivery, thus:

If $\mathrm{T}_{\text {delivery }}<1 \mathrm{~h}$ we start delivery at 8 to avoid congestion

Else $_{\text {departure }}=8 \mathrm{~h}-$ VHT $^{\mathrm{i}}{ }_{\mathrm{w}, \mathrm{K}-1}$

$\mathrm{VHT}_{\mathrm{w}, \mathrm{K}-1}^{\mathrm{i}}$ : vehicle hour traveled to deliver the client $\mathrm{i}$ from the warehouse during the time interval K-1.

Parameters of refrigerated vehicles are shown in table 1.

Based on the mileage and the estimated VHT, costs and carbon emissions were measured and compared to those in normal conditions.

Table 1. Vehicle parameters

\begin{tabular}{l|l}
\hline parameter & value \\
\hline Load capacity $(\mathrm{kg})$ & 795 \\
Fuel type & gasoline \\
maximum speed $(\mathrm{km} / \mathrm{h})$ & 120 \\
fuel consumption when loaded $(\mathrm{km} / \mathrm{l})$ & 2,65 \\
fuel consumption when empty $(\mathrm{km} / \mathrm{l})$ & 6,06 \\
\hline
\end{tabular}

\subsection{Results and analysis}

In this section, we will illustrate experimental results. The VHT has evidently increased during rush hours. This augmentation did not affect customers in scenario A.1, since the demand can be delivered in agreed time. However, in the scenario A.2, the time is violated which leads to additional penalty costs and impacts customer satisfaction. In this case, the time window agreed with these customers should be reviewed to be less restrictive. Another way is to limit the reception of demands to a specific period less congested, but this solution is not practical since we are dealing with restaurant that needs 
food in a precise time so they can process and serve fresh food to customers (breakfast, lunch...).

The fuel consumption is time-dependent; hence its heavy increases in the scenario A.2.

As we are delivering perishable foods, refrigeration costs are a very important component that should be considered. Regarding the latter, we can say that congestion has a deeply negative impact since even for a slight increase on the VHT, the costs increased by an average of $30 \%$.

Since the co2 emissions are directly related to the fuel consumed, it remains' relatively flat as the trip length and the travel time increase.

In the direct delivery, we have demonstrated that fuel consumption increases with the VHT, however

\section{Scheduling and time windows extension}

Through the analysis of different scenarios, we have proved that travel time during rush hours is longer than in other periods, which leads to additional cost, impact the service quality and increases the $\mathrm{co} 2$ emission. With strict time windows, businesses have difficulties in optimizing these costs.

These findings imply that carriers should reduce travel time. After showing how the departure time of each vehicle from the distribution center affects costs and

Service, this goal can be achieved by changing route start times to avoid congested times and traveling as fast as is allowed by the traffic conditions, and combined with more flexibility in delivery time windows.

For this purpose, we have developed two algorithms to schedule departure in the direct delivery scenario and the LTL delivery while extending the time window. To discover how these changes in policy may lead to cost saving.

\section{1. scheduling algorithm}

To plan the departure of the vehicle from the distribution center, we have developed two algorithms for each scenario (direct delivery and LTL). These algorithms are used when the demand is received during a rush hour followed by a time interval with less congestion. The concept consists of programming the departure as late as possible to avoid congestion and travel as fast as possible while respecting the time windows.

\subsubsection{Scheduling technique for scenario $A$}

The demand is received during the time interval $\mathrm{K}$, let Tw be the length of time windows. For a delivery from the distribution center ' 0 ' to a customer ' $\mathrm{n}$ ', the departure is programmed as follow:

$$
\begin{aligned}
& \text { 1: If } V H T_{0}^{n}+\left(T_{k \sup }-T D P\right)<T W \\
& \text { 2: } D_{0}^{n}=T_{k \sup } \\
& \text { 3: Else }
\end{aligned}
$$

strangely we have noticed in scenario B that with a slight increase in VHT the fuel consumption is less under congestion than under normal traffic conditions in some arcs of the tour and consequently, the carbon emissions are reduced because the travel time saved is not really important. In these arcs it is not really wise to speed up for saving 1 or $2 \mathrm{~min}$ in the travel time.

These findings give us an idea about the optimal speed in each segment of the tour. But since we have another crucial component related to the VHT that should be considered while deciding either the speed is advantageous or not. For this reason, we have analyzed the impact on refrigeration cost, and we have found that congestion has a strong impact. It increases costs with an average of $37 \%$ on each tour trip.

$$
\begin{aligned}
& k+1 \\
& 4: V H T_{0}^{n}=T W_{\text {sup }}-T_{k \text { sup }} \\
& 5: D_{0}^{n}=T_{k \sup }-\frac{D_{r}}{V_{k}} \\
& k+1 \\
& 6: D_{r}=V K T_{0}^{n}-V H T_{0}^{n} \times V_{k+1}
\end{aligned}
$$

\subsubsection{Scheduling technique for scenario $B$}

The procedure starts by setting the arrival date to the last customer in the route to the upper bound of the time windows. The departure from the upstream customer is then calculated figure 7 .

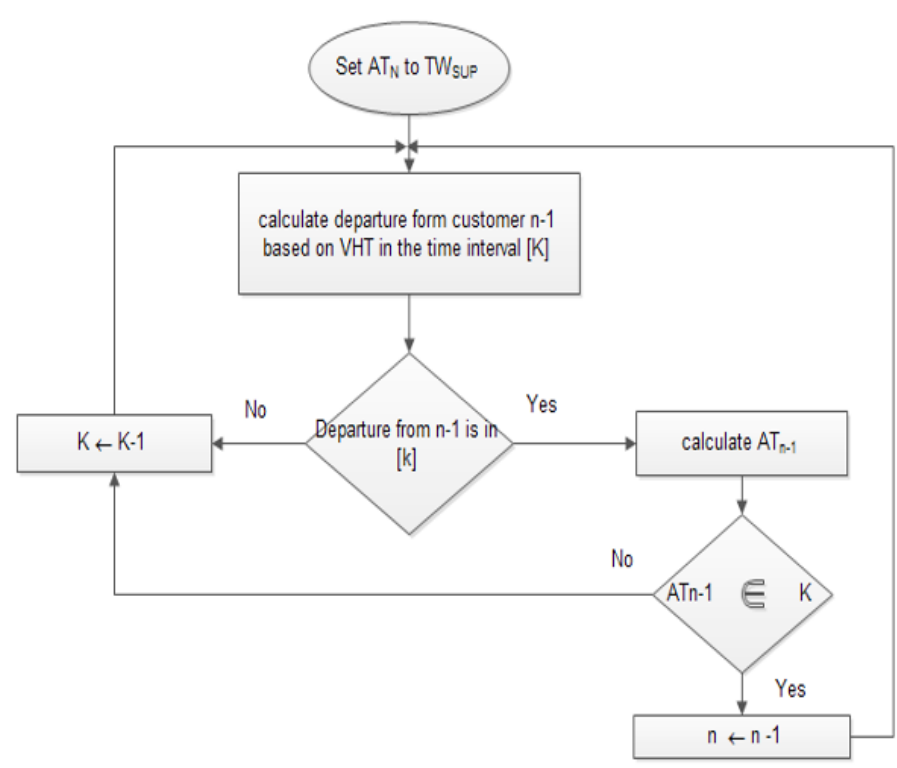

Figure 7. scheduling procedure for scenario $\mathrm{B}$

\subsection{Experience and results}

To validate the proposed algorithms, they were applied to plan departures for scenario A and B.

In the scenario $A$, the algorithm is tested to determine the departure from DC to customer 5, which is critical as shown in our previous analyses. The time window is extended by $15 \mathrm{~min}$. In the scenario B, we 
extend the time windows by 1 hour and we plan departures for 2 vehicles serving the customers in 2 tours.

Experimental results have proved the efficiency of these policy changes in saving costs and reducing emission. Interestingly, for a slight delay of the departure in the scenario A, fuel consumption as well as the co2 emissions have been reduced by $12 \%$ and refrigeration cost by $19 \%$.

In the scenario $\mathrm{B}$, policy changes lead to good results, since the travel time was decreased by $20 \%$ even if the improvement in terms of costs is negligible.

\section{8 conclusions}

This study aims to solve one of the major problems encountered by carriers, which consist of choosing the optimal routes and program departure to deliver customers intime. Therefore, agent-based simulation combined with GIS is used. Simulation runs were performed to estimate the VKT and VHT. Analyses of network changes (congestion) were conducted to provide insight into the impact of these changes on these 2 values (VHT and VKT) also on cost, service quality, and emissions.

A time-dependent scheduling technique was developed and proved its success in achieving a potential saving, in terms of costs and co2 emission. As a future research, this time-dependent vehicle routing problem will be represented as an optimization model and integrated with ABM-GIS.

\section{Acknowledgment}

The first author is supported by the national center for scientific and technical research in Morocco (CNRST) through an excellence scholarship.

\section{References}

1. C. I. Hsu \& W. T. Chen. Optimizing fleet size and delivery scheduling for multi-temperature food distribution. Applied Mathematical Modeling, 38(3), 1077-1091, (2014).

2. N. A. Gómez-Cruz, I. Loaiza Saa, \& F. F. Ortega Hurtado. Agent-based simulation in management and organizational studies: a survey. European Journal of Management and Business Economics, 26(3), 313328 , (2017).

3. P. B.Keenan. Spatial decision support systems: a coming of age. Control and Cybernetics, 35(1), 9, (2006)

4. C. D.Tarantilis, \& C. T. Distribution of fresh meat. Journal of Food Engineering, 51(1), 85-91, (2002)

5. Y. W.Chen, C. H., Wang, \& S. J. Lin. A multiobjective geographic information system for route selection of nuclear waste transport. Omega, 36(3), 363-372, (2008).

6. M.Sharifi,M. Hadidi, E. Vessali, P. Mosstafakhani, K. Taheri, S.Shahoie, \&M. Khodamoradpour. Integrating multi-criteria decision analysis for a GISbased hazardous waste landfill sitting in Kurdistan
Province, western Iran. Waste management, 29(10), 2740-2758, (2009).

7. A. V. Bhambulkar. Municipal solid waste collection routes optimized with arc gis network analyst. International Journal Of Advanced Engineering Sciences And Technologies Vol, (11), 202-207, (2011).

8. M. A. Figliozzi. Analysis of the efficiency of urban commercial vehicle tours: Data collection, methodology, and policy implications. Transportation Research Part B: Methodological, 41(9), 1014-1032, (2007).

9. A. L. Erera. Design of large-scale logistics systems for uncertain environments. University of California, Berkeley, (2000).

10. T. W. Chien. Operational estimators for the length of a traveling salesman tour. Computers \& operations research, 19(6), 469-478, (1992).

11. O.Kwon, B. Golden, and E. Wasil. Estimating the length of the optimal TSP tour: an empirical study using regression and neural networks. Computers \& operations research, 22(10), 1039-1046, (1995).

12. L. Schipper, M. Cordeiro, \& W. S. Ng. Measuring the carbon dioxide impacts of urban transport projects in developing countries. In Proceedings of the Transportation Research Board Conference. Washington, DC. USA. (2007, November) 